\title{
Besser keine konstanten Grenzwerte verwenden
}

- Ohne Spirometrie keine Diagnose einer Atemwegserkrankung, betonte Professor Dr. Helgo Magnussen vom Krankenhaus Großhansdorf beim PneumoUpdate 2009 in Wiesbaden. Allerdings gibt es dabei Fallstricke. Bei der Messung der
Vitalkapazität (VC) während der forcierten Expiration, wie bisher noch empfohlen, wird eine COPD bei gesunden Älteren überdiagnostiziert, sagte Magnussen. Er empfiehlt stattdessen die Messung der VC während der Inspiration. Zudem

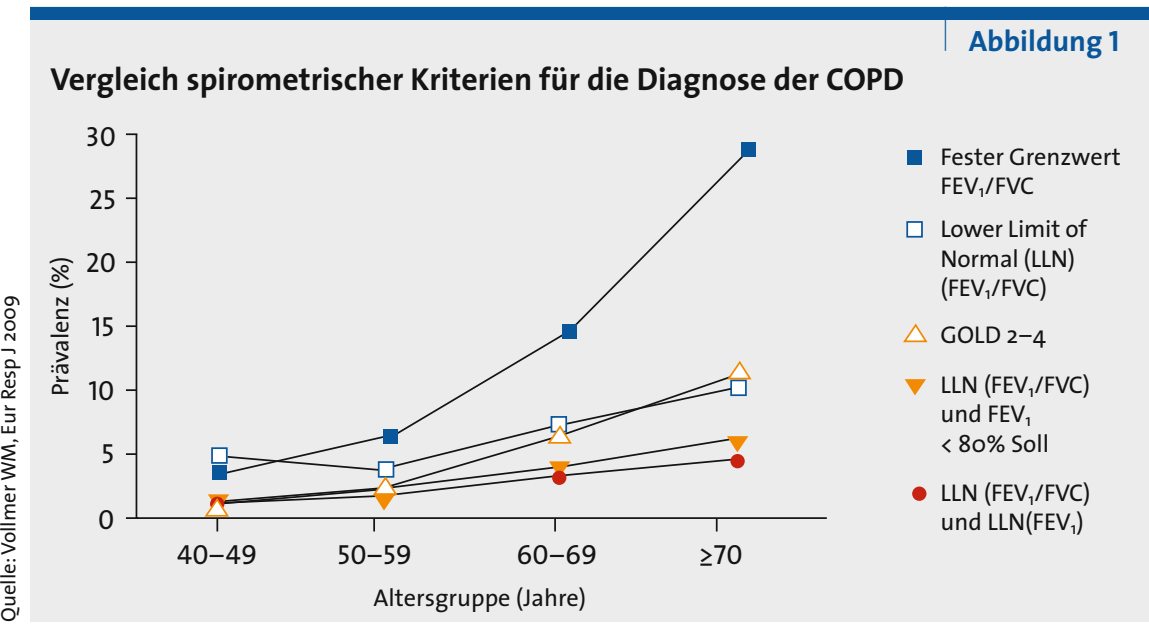

Wird für $\mathrm{FEV}_{1} / \mathrm{FVC}$ ein fixer Grenzwert definiert, kommt es bei älteren Menschen zu einer COPD-Überdiagnostik. sollte kein Cut-off-Wert für das Verhältnis $\mathrm{FEV}_{1} / \mathrm{FVC}$ festgelegt werden, da der Wert stark altersabhängig ist und beim üblichen Grenzwert kleiner 70\% die Rate pathologischer Werte ab dem Alter von 70 Jahren dramatisch ansteigt.

Eine Task Force der European Respiratory Society (ERS) empfiehlt, Werte in der untersten Quintile als pathologisch zu betrachten. Bei Verwendung dieser Werte anstelle des GOLD-Kriteriums $\mathrm{FEV}_{1} / \mathrm{FVC}$ wird die Überschätzung einer COPD im fortgeschrittenen Alter vermindert und die klinisch relevante irreversible Atemwegsobstruktion besser vorhergesagt, so Magnussen.

\section{Fehlerquelle auch bei Asthma bronchiale}

Auch bei der spirometrischen Diagnostik bei Patienten mit Asthma bronchiale sollten keine konstanten Cut-off-Werte benutzt werden. Magnussen: Hier ist das Problem, dass eine Atemwegsobstruktion leicht übersehen wird.

\section{Nachweis pulmonaler Rundherde}

\section{MRT fast so sensitiv wie CT}

- Zum Nachweis pulmonaler Rundherde (PRH) ist das Multi-Detektor(MD-)CT mit einer Schichtdicke von 1 $\mathrm{mm}$ der Standard. Überraschenderweise, so Professor Dr. Gerhard Mostbeck aus Wien beim PneumoUpdate, schnitt das MRT mit einer Schichtdicke von $5 \mathrm{~mm}$ in einer aktuellen Studie bei der Erkennung maligner Herde genauso gut ab.

Für die Vergleichsstudie wurden aus einem Kollektiv von mehr als 400 Patienten 161 Patienten, darunter 73 Raucher, mit insgesamt 200 PRH ausgewählt. Im Schnitt hatten die PRH einen Durchmesser von 15,6 mm, 103 waren maligne und 97 benigne. Die Sensitivität zum Nachweis von Rundherden war beim MD-CT mit 97\% signifikant höher als beim MRT (82,5\%), berichtete Mostbeck. Allerdings zeigte sich beim
Nachweis maligner Herde kein signifikanter Unterschied zwischen beiden Techniken (100\% beim CT vs. 96\%

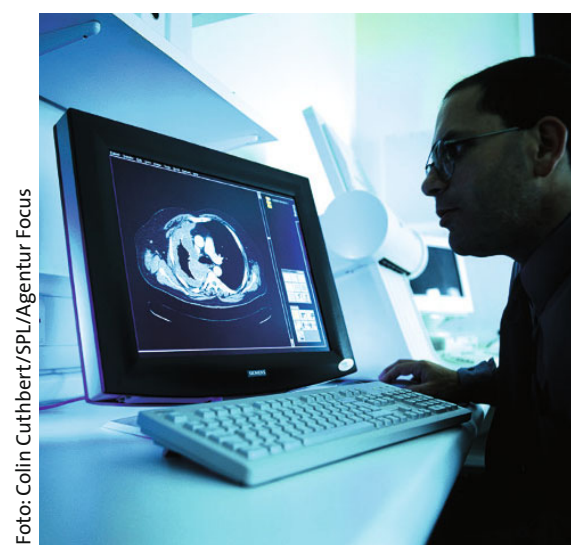

MRT: Für ein Bronchialkarzinom-Screening eine mögliche Alternative zum CT. beim MRT). Von den drei eingesetzten MR-Sequenzen erwies sich die STIRSequenz (im Vergleich zum T1- und T2-gewichteten MRT) als diagnostisch am wertvollsten.

Die etwas ungenauere MRT fokussiert nach diesen Daten bei der Abklärung eines Bronchialkarzinoms eher auf die Erkennung größerer maligner Läsionen. Damit könnte das MRT als Screeningmethode geeignet sein, sagte Mostbeck, insbesondere unter dem Aspekt, dass sich beim Einsatz des CT zum Screening häufig falsch positive Befunde ergeben. Auch wegen der Strahlenbelastung sollte zum Beispiel bei schwangeren Patientinnen oder bei jungen Patienten, bei denen mehrere Verlaufsuntersuchungen absehbar sind, alternativ zur CT an das MRT gedacht werden. 